\title{
A new generation of Solid Phase Microextraction coatings for complementary separation approaches: a step toward comprehensive metabolomics and multiresidue analyses in complex matrices
}

\author{
Emanuela Gionfriddo, Ezel Boyac1, and Janusz Pawliszyn* \\ Department of Chemistry, University of Waterloo, Waterloo, Ontario, Canada
}

\begin{abstract}
In this work, a new generation of SPME coatings based on polytetrafluoroethylene amorphous fluroplastics (PTFE AF 2400) as a particle binder is presented. The developed coating was tested for thermal and solvent-assisted desorption, demonstrating its compatibility with both gas- and liquid-chromatographic platforms. The incorporation of hydrophilic-lipophilic balance (HLB) adsorptive particles provided optimal extraction coverage for analytes bearing a broad range of hydrophobicities, molecular weights, and of varied chemical diversity. The performance of the newly developed coating was compared to already established coatings based on different polymers such as DVB/Car/PDMS and C18/SCX/PAN in order to assess the new prototype versus the existing technology. As this is the first documented instance of PTFE AF being used as a particle immobilizer for SPME, an assessment of the analyte uptake rate and extraction capability of the developed coating was carried out in comparison to other conventionally used polymers. Moreover, the new SPME probes were used to validate an analytical method for determination of banned doping substances, achieving limits of quantitation below the minimum required performance limits (MRPLs) set by the World Anti-Doping Agency (WADA) for most compounds. Considering the broad coverage of the coating in terms of analytes extracted, and its suitability for both thermal- and solvent-assisted desorption, these new SPME probes will properly suit various metabolomics applications that involve the use of both gas- and liquid-chromatography.
\end{abstract}

The importance of microsampling tools that allow for realtime characterization of samples and objects including living systems has grown exponentially over the last two decades. Solid-phase microextraction (SPME) represents to date one of the most versatile sample preparation techniques, and its use in bioanalytical, food, and environmental analysis has gained widespread acceptance. ${ }^{1-3}$ A critical characteristic of a given microsampling device is its extraction phase, which must be tailored to specific applications under consideration. Consequently, substantial attention has been given to the development and commercialization of a wide array of coatings bearing different chemistries and geometries, a practice that continues to expand to date as the use of microsampling further extends towards different areas of research. A significant body of research has validated the reliability of SPME tools toward targeted, multi-residue, and untargeted analysis; further, SPME extracting phases able to extract a very broad ranges of analytes characterized by vast physical-chemical diversity are readily available today owing to significant advances achieved in in-vivo studies and metabolomics investigations. ${ }^{4}$ For metabolomics applications, in particular, the amount of chemical information retrieved from the studied system plays a critical role in the outcome of the obtained results. Thus, a combination of different chromatographic platforms would properly serve this purpose, allowing for the collection of a broader range of analytes. ${ }^{5}$ In light of this, an SPME coating that can be properly tailored for thermal and solvent desorption would be the best option for extraction of analytes amenable to gas chromatography (GC) and liquid chromatography (LC). The availability of such a microsampling probe would undoubtedly expand the number of chemical entities extracted, which in turn would allow for a more comprehensive description of the metabolome of the system under investigation. To date, SPME coatings are mainly constituted by different sorptive particles immobilized by polymers of different natures that contribute to the extractive process and enhance coating compatibility toward complex matrices. ${ }^{6,7}$ Generally, coatings based on polyacrylonitrile (PAN), mainly used for solvent-assisted desorption in LC applications, are incompatible with thermal desorption conditions used during GC analysis due to the thermal degradation of PAN. ${ }^{8}$ Conversely, polydimethylsiloxane (PDMS) based coatings, while capable of withstanding typical thermal desorption temperatures, usually present bleeding peaks, may swell when in contact with certain solvents, and are characterized by a preparation procedure that requires a long curing process. In recent years, fluorocarbon polymers have found many applications in analytical chemistry for extraction and transport purposes. ${ }^{9,10}$ Among the variety of fluoropolymers available, 
polytetrafluoroethylene amorphous fluoropolymers (PTFE AFs) represent one of the most studied classes of perfluoropolymers due to their suitability toward various applications, such as chemical separations, sensors, and bioassay platforms. ${ }^{11}$ Outstanding work carried out by Weber's group aimed at the elucidation of the properties of these materials as media for extraction and transport. ${ }^{12-16}$ In view of their physicochemical properties, such us high thermal resistance and inertness towards most organic solvents, PTFE AFs can be considered good candidates as particle immobilizer media for solid-phase microextraction devices. In addition, the use of these polymers would enhance the biocompatibility of the SPME coating, ${ }^{17}$ extending its applicability to direct analysis of complex matrices such as biofluids and foodstuffs. With the aim of creating extraction phases suitable for untargeted metabolomics studies, hydrophilic-lipophilic balance (HLB) particles constitute the best choice of sorbent in view of their ability to efficiently extract a broader range of analytes in comparison to other commonly used SPE and SPME sorbents. ${ }^{18-20}$ In this work, the development of a new fluoropolymer/ hydrophilic-lipophilic balance (PTFE AF/HLB) coating is presented, and its suitability for extraction of a broad range of analytes, as well as its compatibility with thermal and solvent-assisted desorption are demonstrated. To the best of the author's knowledge, this is the first instance to date where HLB particles glued together by a fluorocarbon-based polymer have been applied as an extraction phase for SPME coupled. Satisfactory results were obtained in terms of coating extraction capability, reproducibility, limits of detection, and quantification in comparison with commercially available GCand LC- compatible SPME coatings. In addition, a preliminary evaluation of coating biocompatibility was conducted in whole blood samples.

\section{EXPERIMENTAL SECTION}

Material and Chemicals. The properties of the GC- and LCamenable analytes under study are provided in Table S1 (Supplementary Information) together with corresponding supplier information. The LC-MS grade solvents water $\left(\mathrm{H}_{2} \mathrm{O}\right)$, acetonitrile $(\mathrm{ACN})$, and methanol $(\mathrm{MeOH})$ were purchased from Fisher Scientific (Ottawa, ON, Canada) and used for chromatographic separation and preparation of desorption solutions. Formic acid (FA), mass spectrometry grade, was obtained from Fluka (Oakville, ON, Canada) and used as an additive in the mobile phases. Commercial SPME fiber assemblies in 23-gauge needle sizes, namely, 50/30 $\mu \mathrm{m}$ DVB/Car/PDMS (stableflex), were purchased from Supelco (Oakville, ON, Canada). Mixed-mode fibers (C18/SCX/PAN) were kindly provided by Supelco (Bellefonte, PA, USA). Mixed-mode coatings of these fibers are constituted by silica particles bonded with C18/benzenesulfonic acid, with a $1.5 \mathrm{~cm}$ coating length at an average thickness of $45 \mu \mathrm{m}$. The evaluation of fibers for LC analysis was performed in a phosphate-buffered saline (PBS) solution with $\mathrm{pH}$ adjusted to 7.4. Salts used for preparation of PBS, namely sodium chloride, potassium chloride, potassium phosphate monobasic, and sodium phosphate dibasic were all purchased from Supelco (Oakville, ON, Canada). HLB $5 \mu \mathrm{m}$ particles were kindly provided by Waters. PTFE AF 2400 was purchased from Sigma-Aldrich (Oakville, ON, Canada ), FC-72 ${ }^{\mathrm{TM}}$ was obtained from Acros Organics-Fisher Scientific. A stock solution containing all analytes under study was prepared by appropriate dilutions of each individual standard in $\mathrm{MeOH}$, resulting in a final concentration of $20 \mu \mathrm{g} \mathrm{mL} \mathrm{m}^{-1}$. Working solutions were prepared daily in PBS by spiking the required amount from the mixed stock solution. Mobile phases were degassed for 20 min in a VWR Scientific, Aquasonic model 75HT (West Chester, PA, USA) ultrasonic bath before use. The $\mathrm{pH}$ level of all prepared solutions was measured using a Metter Toledo MP220 (Schwerzenbach, Switzerland) $\mathrm{pH}$ meter. When evaluating the coatings for GC analysis, individual stock solutions $\left(2.5 \mathrm{mg} \mathrm{mL}^{-1}\right)$ of each analyte were prepared in methanol and acetone. A stock mixture standard solution and subsequent working dilutions were prepared in methanol and stored in a freezer at $-30^{\circ} \mathrm{C}$. The concentration of each analyte in the working mixture was carefully adjusted in order to provide an acceptable response for all investigated analytes. The samples were freshly prepared as needed by spiking an appropriate amount of working solutions in nanopure water $(18.3 \mathrm{M} \Omega \mathrm{cm})$, obtained from a NANOpure water system from Barnstead International, Dubuque, USA.

LC-MS instrumentation. The LC-MS system used for the study consisted of an Accela ultra high-pressure liquid chromatograph (UHPLC) pump equipped with an Accela autosampler, and connected to an Exactive ${ }^{\mathrm{TM}}$ benchtop Orbitrap mass analyzer, all of which was provided by Thermo Scientific (San Jose, CA, USA). Additional details on separation conditions and mass spectrometric conditions can be found in Supporting Information (Section 1).

GC-MS instrumentation. An Agilent 6890/5973 GC-MS equipped with a GERSTEL CIS septumless PTV injector and with a CIS deactivated glass liner for SPME (I.D. 1mm), and a GERSTEL MultiPurpose Sampler MPS were used. Further details are provided in Supporting Information (Section 2).

SPME experiments. In order to assess the performance of the coating for LC applications, various parameters were investigated, such as optimum desorption solvent (Supplementary Information Section 3), extraction and desorption time profiles, limits of quantification (LOQ), and inter-fiber and intra-fiber reproducibility. All experiments were performed in triplicate using a multi-tube vortexer from Fisher Scientific (USA). A typical experiment consisted of conditioning, pre-extraction washing, extraction, postextraction washing, and desorption steps. Prior to extraction, fibers were conditioned for $15 \mathrm{~min}$ at $1200 \mathrm{rpm}$ agitation speed in $1.8 \mathrm{~mL}$ of an $\mathrm{ACN} / \mathrm{MeOH} / \mathrm{H}_{2} \mathrm{O}(40 / 40 / 20, \mathrm{v} / \mathrm{v} / \mathrm{v})$ mixture acidified to contain $0.1 \%$ FA. This step serves not only to wet the bonded functional groups of the coating but also as a further desorption step to remove potential carry-over from preceding experiments. The conditioning step was always followed by a quick rinsing (2 s) of the coating in 1.8 $\mathrm{mL}$ of water. This step is useful to avoid introduction of any residue of organic solvent (eventually present on the coating after conditioning) into the sample. Subsequently, extraction experiments were performed in $1.8 \mathrm{~mL}$ of PBS at $1200 \mathrm{rpm}$ agitation speed. Following each extraction, a 2 s static washing step employing $0.1 \mathrm{~mL}$ of water was carried out. Finally, desorption of analytes from the fiber was 
accomplished using $0.1 \mathrm{~mL}$ of $\mathrm{ACN} / \mathrm{MeOH} / \mathrm{H}_{2} \mathrm{O}(40 / 40 / 20$, $\mathrm{v} / \mathrm{v} / \mathrm{v})$ acidified with FA $(0.1 \% \mathrm{v} / \mathrm{v})$ as desorption solution, with sample agitation set at $1200 \mathrm{rpm}$ for $30 \mathrm{~min}$. To assess the performance of the coating for GC applications, direct immersion extractions were performed in $9 \mathrm{~mL}$ of ultrapure water spiked with the analytes of interest. The sampling conditions were: $30 \mathrm{~min}$ extraction at $30^{\circ} \mathrm{C}$, and $500 \mathrm{rpm}$. Analyte desorption was carried out in the GC injector port for $15 \mathrm{~min}$ at $270^{\circ} \mathrm{C}$. Consecutive fiber blanks of the fiber did not reveal the presence of analyte carry-over on the coating, thus confirming the efficiency of the desorption conditions used.

Coating preparation optimization. The optimization of the coating preparation procedure represents the most critical step in obtaining a suitable coating with proper thickness, robustness, and surface homogeneity.A $127 \mu \mathrm{m}$ diameter stainless steel wire was electro-etched by applying a voltage of $3.5 \mathrm{~V}$ for $30 \mathrm{sec}$, in order to achieve proper degree of roughness necessary to guarantee robust adhesion of the extraction phase. Afterward, the wire was thoroughly washed with deionized water to remove excess salt, and sonicated in methanol for 10 minutes. Preparation of the extraction phase coating consisted of mixing a fluorocarbon-based fluid (FC72) with $5 \mu \mathrm{m}$ HLB Oasis ${ }^{\circledR}$ particles $(2.6 \% \quad w: w)$ and sonicating for 10 minutes. Following, PTFE AF 2400 was added to the particle suspension in a ratio of 1:1 w: $\mathrm{w}$ (PTFE $\mathrm{AF} / \mathrm{HLB}$ ), and vortexed overnight. The coating procedure was carried out by consecutive dipping of the stainless steel core into the above described slurry. After each dipping cycle the coating was left to dry at room temperature for $1 \mathrm{~min}$ to remove the solvent. One of the main advantages of this procedure in relation to the specific materials used for coating preparation lies in the possibility of easily tailoring the coating thickness by use of successive dipping cycles, avoiding the need for time-consuming curing steps.

- The optimization of the coating procedure resulted in adequate coating features, such as homogeneity, as illustrated in Figure 1. The abovementioned coating was successively used for thermal desorption applications, and its performance compared to that of the commercial DVB/Car/PDMS coating of similar thickness $(50 \mu \mathrm{m}$ DVB layer and $30 \mu \mathrm{m}$ Carboxen (B) layer). In the case of solvent-assisted desorption, the performance of the PTFE AF/HLB coating was compared to a C18/SCX/PAN coating (45 $\mu$ m thickness) used especially for metabolomics applications with liquid chromatography. In this case, a new coating of $\sim 20 \mu \mathrm{m}$ thickness was prepared (Figure S1). Scanning electron microscopy (SEM) images were acquired with the use of a LEO 1530 field emission (Carl Zeiss NTS GmbH, Germany). An Olympus SZX10 stereomicroscope system equipped with an SC30 digital camera (Olympus, Japan) was used for acquisition of microscopic images. Thermal gravimetric analysis (TGA) was carried out using a Q600 SDT (TA Instruments).

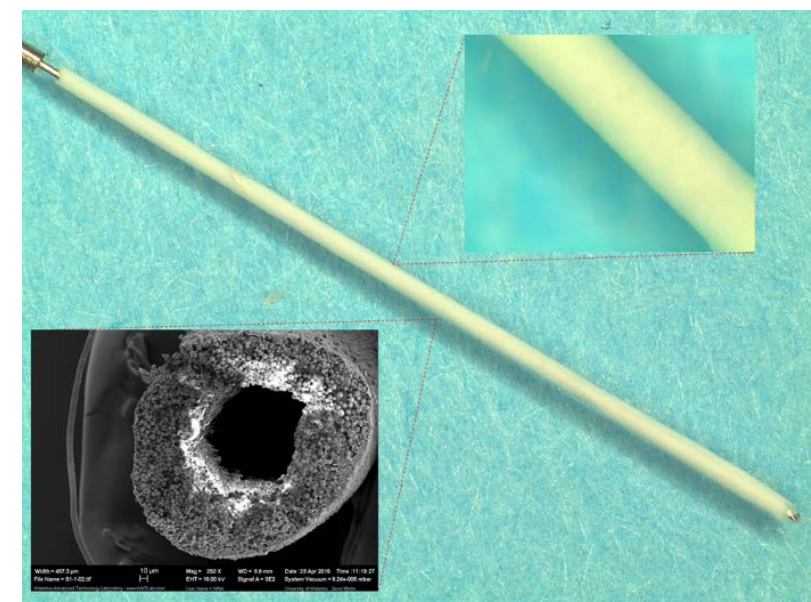

Figure 1. PTFE AF/HLB coating obtained with the use of the dipping procedure. The calculated thickness was $\sim 90 \mu \mathrm{m}$, obtained through 7 consecutive dipping cycles.

\section{RESULTS AND DISCUSSION}

Evaluation of coating performance by thermal desorption. Initially, coating performance was tested under thermal desorption conditions to evaluate the suitability of the coating for GC applications. The thermal stability of the extraction phase was verified by TGA (Figure S2). The TGA curve shows only a $1.4 \%$ decrease in total weight between $50-300$ ${ }^{\circ} \mathrm{C}$, which is mainly associated with loss of adsorbed water $\left(50-100{ }^{\circ} \mathrm{C}\right)$ and absorbed water $\left(100-250^{\circ} \mathrm{C}\right)$, and some early indications of polymer-related losses $\left(250-300{ }^{\circ} \mathrm{C}\right)$. The main weight loss, which is directly associated with the thermal decomposition of the polymer, occurs after $300{ }^{\circ} \mathrm{C}$, indicating that the polymer is sufficiently stable for thermal desorption. However, it is important to emphasize that TGA analysis can only provide eventual decomposition trends under gradual and short-termed temperature changes, and hence, cannot supply information concerning the effect of longer and continuous exposure to constant temperatures. In light of this, further tests were performed to test the thermal stability of the coating, as described below. Coating stability was tested by conditioning the fiber at $200^{\circ} \mathrm{C}$ for one hour under inert gas flow $\left(\mathrm{N}_{2}\right)$. The coating was then submitted to inspection via microscope, revealing no alterations to its surface morphology. Further conditioning at $250^{\circ} \mathrm{C}$ (typical temperature used for commercial SPME coating desorption) under the same conditions was performed, with subsequent inspection via microscope indicating coating morphology was not undermined. The use of thermal blanks did not reveal a significant presence of peaks due to bleeding of the coating. Minor peaks related to divinylbenzene and $\mathrm{N}$-vinylpyrrolidone derivatives were detected, likely stemming from HLB particles; however, this is not an uncommonly observed phenomenon for other types of polymers constituting SPME commercial coatings ${ }^{21-23}$. Thus, it does not constitute a major concern for the chemical integrity and correct functioning of the extraction phase. Subsequently, the coating was subjected to a series of $30 \mathrm{~min}$ extractions in ultra-pure water spiked with a mixture of 11 analytes (benzene, 1-pentanol, 2hexanone, ethyl butanoate, $\alpha$-pinene, benzaldehyde, eucalyptol, acetophenone, 2-undecanone, ethyl nonanoate and 1-pentanol) at different concentrations ranging in the low $\mu \mathrm{g}$ 
$\mathrm{L}^{-1}$ level. Preliminary results, shown in the chromatogram presented in Figure S3, revealed satisfactory extraction performance and efficient analyte desorption $\left(250^{\circ} \mathrm{C}\right.$ for 10 min). In order to assess the performance of the coatings under study, a new mixture of analytes was considered: 23 compounds already identified as fruit metabolites and bearing a broad range of polarities, molecular weights, and functionalities were selected for analysis, and their concentrations accurately adjusted to guarantee proper sensitivity. This selected group of analytes was then used to determine coating extraction capability and applied towards a study of the adsorption dynamics of the coating under kinetic conditions. The performance of the new PTFE AF/HLB coating was compared to that of the DVB/Car/PDMS coating, which to date allows for the extraction of the broadest range of analytes, and has already shown its superiority among other commercial coatings in metabolomics applications. ${ }^{24}$

The coating capacity, expressed as fiber constant $\left(f_{c}\right)$, was calculated with use of Equation 1:

$$
f_{c}=\frac{n_{e} V_{s}}{V_{s} C_{o}-n_{e}}
$$

Where $n_{e}$ is the amount extracted from the coating at equilibrium conditions, $\mathrm{V}_{\mathrm{s}}$ is the sample volume, and $\mathrm{C}_{\mathrm{o}}$ is the original concentration of the analyte in the sample. ${ }^{25}$ The determination of fiber constants is particularly useful in this case, since it includes the term related to the total active surface involved in the extraction, $f_{c}=K_{f s} \times S_{a}$. Thus, no corrections related to different particle sizes or coating volumes were needed in comparisons of different solid SPME coatings. The obtained results for the $\mathrm{f}_{\mathrm{c}}$ of PTFE AF/HLB and
DVB/Car/PDMS coatings, calculated under equilibrium conditions for both coatings (8 $\mathrm{h}$ extraction), are shown in Figure 2. Note that the values provided in the figure were adjusted in order to properly fit the scale of the figure by various multiplication factors. Statistical analysis (Student's ttest) was performed on the obtained values to evaluate if the results provided by both coatings revealed any statistical significant difference (Table S4). The PTFE AF/HLB coating shows improved performance for polar aliphatic molecules such as 1-pentanol, 2-hexanone, hexanal and ethyl butanoate, as well as terpenes such as linalool, eucalyptol and $\alpha$-pinene. Significant differences were found for o-vanillin, carvone, geranial, and neral, benzaldehyde and limonene for which the DVB/Car/PDMS coating shows higher affinity. For the remaining compounds, the calculated values of $f_{c}$ did not present statistical significant differences. Particularly, it is worth noting the extraction capability of the new coating towards molecules bearing the same functional groups but different saturation levels of $\mathrm{CH}$-based moieties (e.g., unsaturated aliphatic vs aromatic), as is the case of hexanal and benzaldehyde, and 2-hexanone and acetophenone. The probed unsaturated aliphatic molecules show higher $f_{c}$ values on HLB sorbent than on the DVB/Car/PDMS coating, while the latter coating shows comparable or better extraction efficiency for aromatic homologs. This phenomenon can be explained by carefully considering the type of interactions occurring between analytes and extraction phase.For solid porous SPME coatings, analyte extraction is influenced by weak intermolecular interactions. ${ }^{26}$

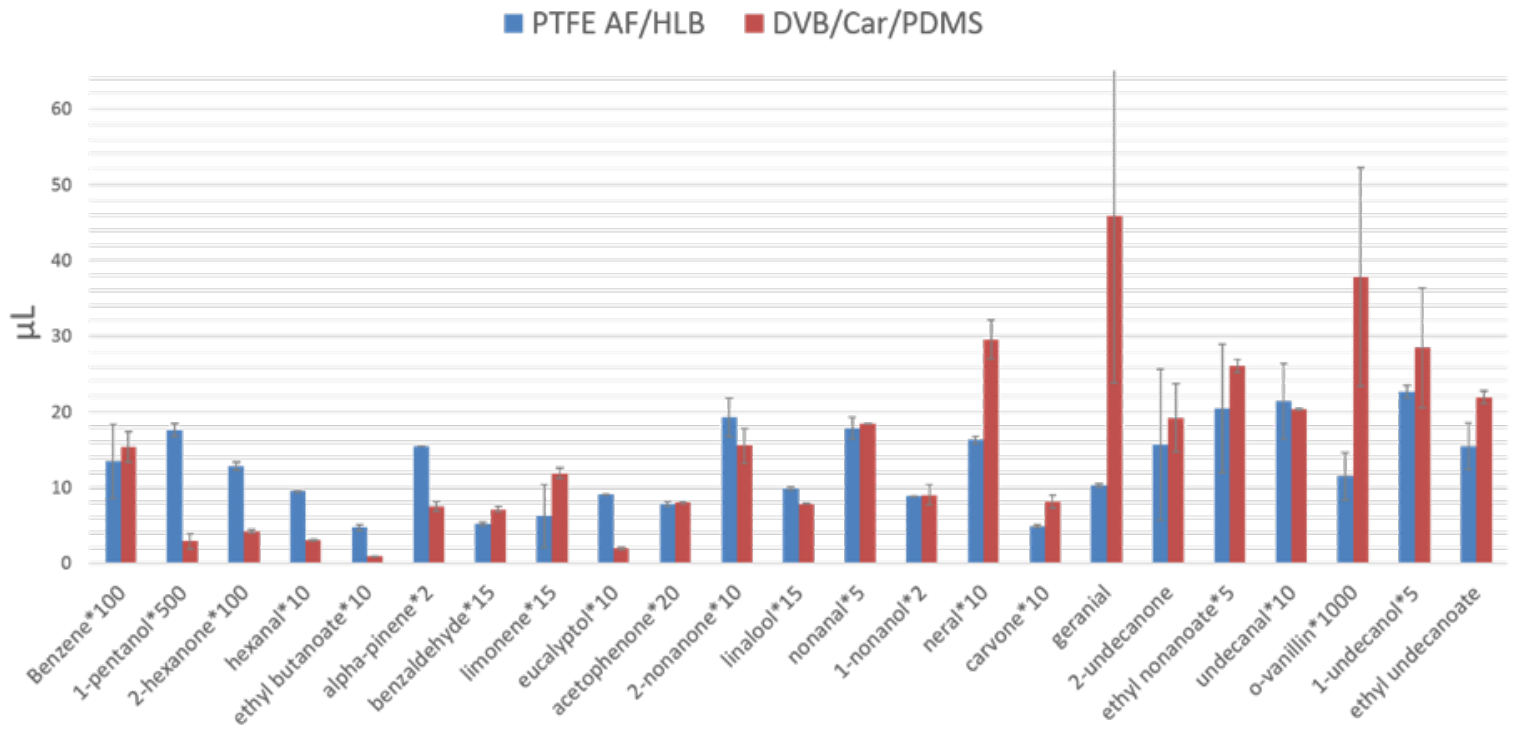

Figure 2. Results obtained for the $\mathrm{f}_{\mathrm{c}}$ of PTFE AF/HLB and DVB/Car/PDMS coatings, calculated under equilibrium conditions for both coatings ( $8 \mathrm{~h}$ extraction). Extractions were performed in aqueous media at a temperature of $30{ }^{\circ} \mathrm{C}$. The values of $f c$ were adjusted to fit the scale of $y$-axis, please refer to individual multiplication factors.

On the surface of the hydrophilic-lipophilic balanced polymeric particles, different functional groups are responsible for the solute/sorbent interactions. Thus, different types of secondary interactions take place $(\pi-\pi, \mathrm{H}$-bonding, Van der 
Waals interactions). In case of short-chain aliphatic molecules such as 2-hexanone and hexanal, the HLB sorbent is capable of interacting with their carbonyl moieties, whereas this kind of interaction is limited on the DVB sorbent, thus resulting in lower extraction efficiency for such compounds. Conversely, when aromatic rings are present, both sorbents provide similar extraction efficiency due to the presence of divinylbenzenederivatives capable of $\pi-\pi$ interactions. It should be noted, the explanations given above only take into consideration interaction between the solute and extraction phase. Subsequently, a careful evaluation of the uptake of the studied analytes on the coating was performed. As this is the first time a fluoropolymer has been used to immobilize particles on an SPME coating, it is important to determine whether the PTFEbased polymer creates a barrier to the mass transfer of the analytes from the sample to the extraction phase, and, in addition, to compare its behavior with PDMS, used for fabrication of commercial coatings. This effect would be particularly evident in instances where adsorption under kinetic conditions is performed. For this purpose, a comparison of the mass uptake rates of the extraction process for both coatings was performed by evaluating approximate trends of the linear region of the extraction time profile (30 s up to $5 \mathrm{~min}$ ) for each analyte ${ }^{27}$. As shown in Figure 3, for molecules bearing high to medium polarity and small molecular weights, the PTFE AF/HLB coating shows a faster analyte uptake compared to the DVB/Car/PDMS coating. On the other hand, for analytes bearing higher molecular weights and low polarities, we can observe in some cases a reversed behavior of the two coatings, where DVB/Car/PDMS provides
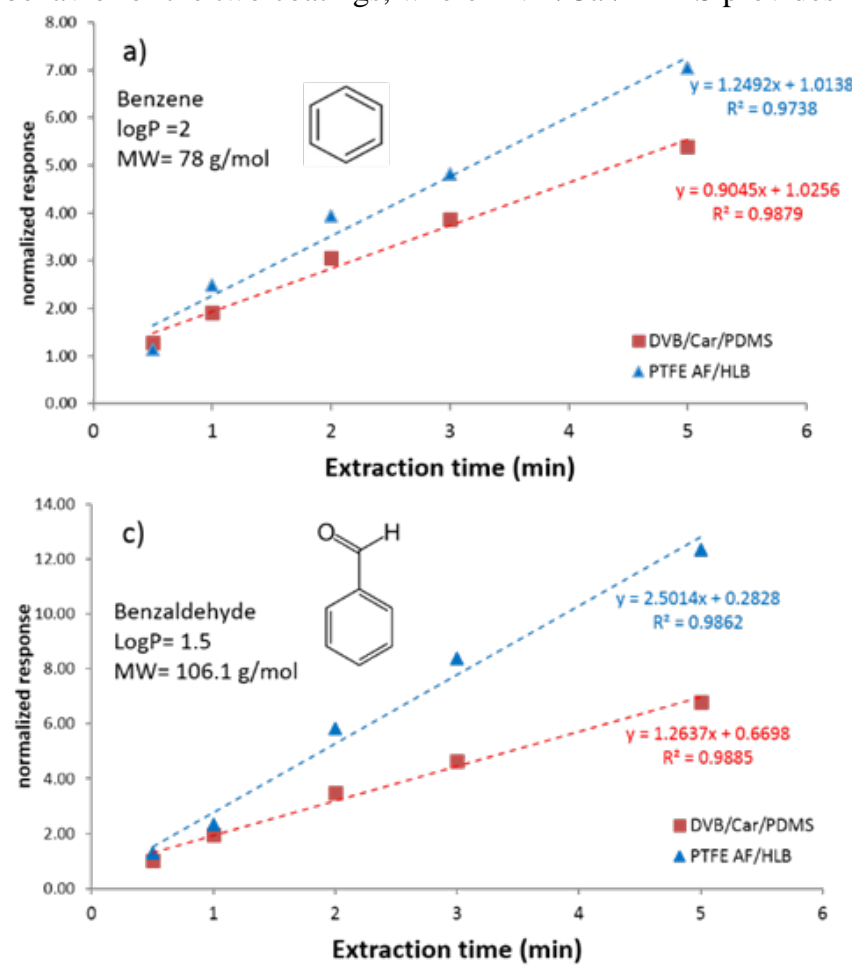

the higher uptake rate, as can be seen in Figures S4 a and c. Conversely, for other unsaturated aliphatic molecules, coating behavior can be said to be similar for the two investigated coatings (Figure S4 b and d). This phenomenon is likely to be dependent on the permeability trends of the two polymers (PTFE AF and PDMS) and their dependence on the critical volumes of the penetrant molecule. ${ }^{28}$ Generally, for glassy polymers such PTFE AF, permeability coefficients decrease as the size of the penetrant increases, while an opposite trend is observed for rubbery polymers such as PDMS, thus resulting in the obtained findings. ${ }^{28}$ The reproducibility obtained for consecutive extractions performed with the PTFE AF/HLB coating showed very satisfactory results, especially when compared to the commercial DVB/Car/PDMS coating (Figure S5). The PTFE AF/HLB coating yielded RSD\% values of less than $5 \%$ for 16 out of the 23 tested analytes. Only 4 compounds were characterized by RSD\% ranging between 5 and $10 \%$, and only three above $10 \%$. Conversely, the commercial DVB/Car/PDMS coating yielded RSD\% values higher than $5 \%$ for 21 out of the 23 tested analytes.

Evaluation of coating performance by solvent desorption. The suitability of any coating to a given SPME-LC application strictly depends on its efficiency in desorbing the analytes quantitatively to a given organic solvent within a reasonable time. Compared to thermal desorption, which is employed for gas-chromatographic applications, the mass transfer of the analytes from the coating to the desorption solvent is slower and in certain cases non quantitative, leading to
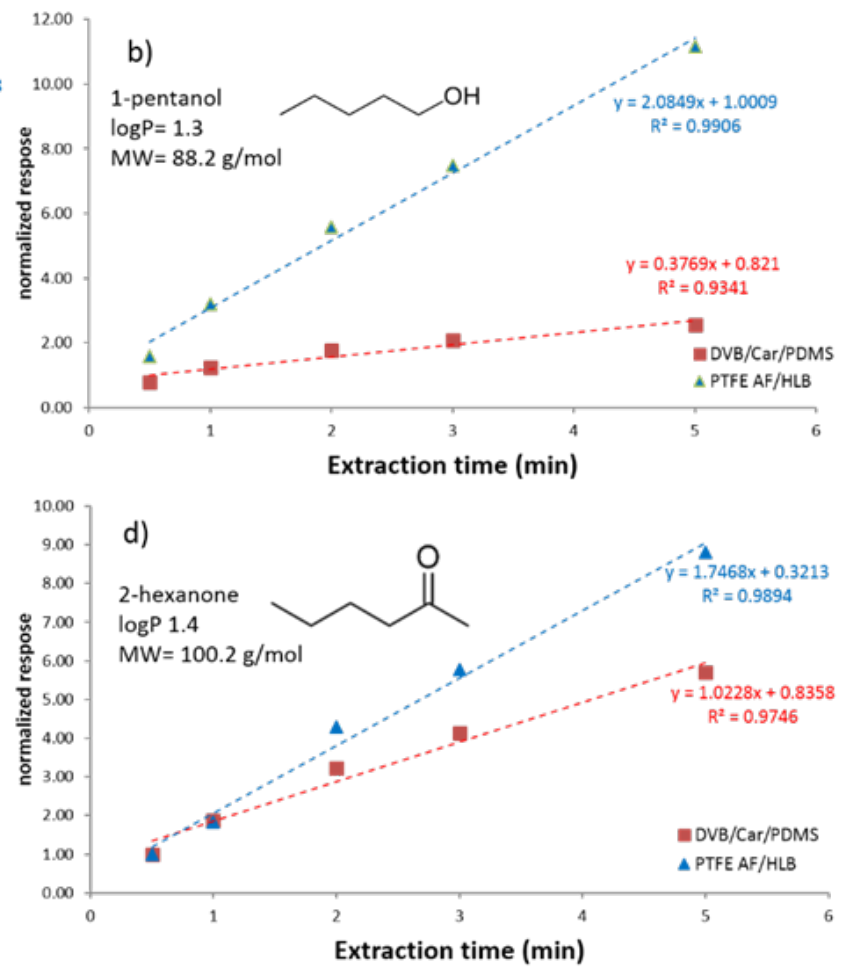

Figure 3. Uptake rate on PTFE AF/HLB and DVB/Car/PDMS for benzene, 1-pentanol, benzaldehyde, and 2-hexanone (representing analytes with high polarities and low molecular weights). Extractions were performed at $30^{\circ} \mathrm{C}$ and $600 \mathrm{rpm}$ in direct immersion mode. 
carry-over effects that bias the outcome of the results. Not all the polymers are suitable for solvent desorption due to their incompatibility with certain desorption solvents and/or the inefficiency and slowness of the desorption process. In this sense, it is already well known that PTFE AF polymers present great stability towards a majority of organic solvents. ${ }^{13}$ Despite this advantage, the possibility that the permeability of the polymer may affect the mass transfer of analytes into and from the coating needs to be investigated. Hence, a careful investigation of the dynamics of extraction and desorption processes as they relate to the set of LC-amenable analytes presented in Table S1 was carried out. In view of the widespread use of C18/SCX/PAN coating for LC-SPME applications owing to the broad range of analytes that they can efficiently extract, ${ }^{29-34}$ these coatings were selected for a comparative description of the characteristics of the novel coating versus existing technology. In addition, an assessment of the uptake rate and coating capacity of the newly developed coating was carried out for LC-amenable analytes. Extraction time profiles of a series of LC-amenable analytes (Table S1) were acquired from $0.5 \mathrm{~min}$ up to $720 \mathrm{~min}$. As this step constitutes the first stage of method optimization, an initial desorption time of $2 \mathrm{~h}$ was chosen to guarantee efficient desorption and avoid carry-over effects (Figure S6). An extraction time investigation showed that most of the compounds under study were noted to reach equilibrium at 360 minutes (with the exception of trenbolone, which yielded a 60 min equilibration time) for PTFE AF/HLB coatings. On the other hand, for C18/SCX/PAN, nikethamide, salbutamol, amphetamine, methamphetamine, budesonide, exemestane, and trenbolone reached equilibrium between 60-120 min. For the remaining compounds evaluated, a plateau of apparent equilibrium was reached within approximately 30-120 min, followed by a further increase of the amount extracted by the coating between 360 and $720 \mathrm{~min}$. The general trend observed is that faster equilibration generally occurs for relatively small compounds, although the exact relationship between equilibration times and the polarities or functional moieties of these compounds is not clear. Overall, both PTFE AF/HLB and C18/SCX/PAN coatings showed similar equilibration times for about $70 \%$ of the compounds under study. In terms of extraction performance, under equilibrium conditions, similar amounts of analyte were extracted in most cases by both coatings. In view of the obtained results, an extraction time of 30 min was selected for further experiments as a compromise between sensitivity and analysis throughput. Following, the performance of the PTFE AF/HLB coating towards solvent desorption was investigated. Different extraction solvents were tested (Supplementary Information Section 3), resulting in the selection of $\mathrm{ACN} / \mathrm{MeOH} / \mathrm{H}_{2} \mathrm{O}$ as the best candidate among the studied solvents in achieving high desorption recoveries and lowest carry-over. Subsequently, a desorption time profile was investigated from 5 to $180 \mathrm{~min}$. The obtained results demonstrated fast kinetics of desorption, with quantitative desorption accomplished within $30 \mathrm{~min}$ for all tested compounds (Figure S7). These results support the suitability of the PTFE AF-based coating for solvent desorption, and thus its applicability for SPME-LC analysis, particularly in view that this desorption strategy had not been reported to date for a coating consisted of PTFE AF. Finally, an evaluation of analyte uptake rate at short extraction times was carried out for both coatings from 0.5 to $5 \mathrm{~min}$, in a fashion similar to that employed for GC analysis. Representative results are shown in Figure 4. From the results in Figure 4 and the abovementioned results, two obvious outcomes were the effects of polarity and of the molecular size of compounds on the relative uptake rate of the fiber coatings. For instance, for polar compounds and small molecules under study, PTFE AF/HLB fibers displayed 

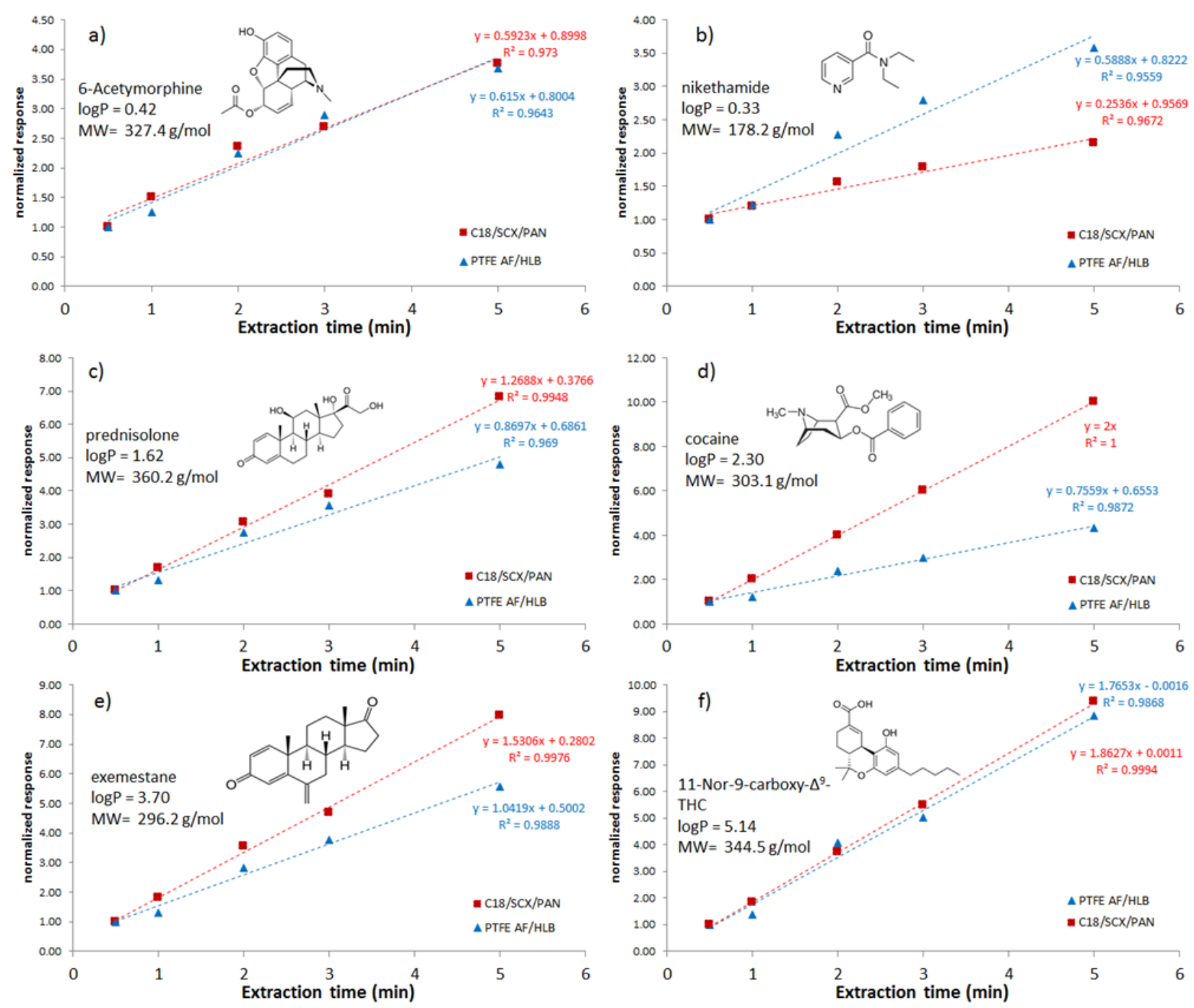

Figure 4. Comparison of uptake rates on PTFE AF/HLB and C18/SCX/PAN coatings.

significantly faster uptake rates than C18/SCX/PAN fibers, while for mid- to nonpolar compounds with relatively larger molecular sizes, the uptake rates of C18/SCX/PAN fibers were faster than those achieved by PTFE AF/HLB fibers. As previously discussed for GC amenable analytes, this phenomenon can likely be attributed to a combination of weak molecular interactions provided by the HLB particles, and the permeability of the analytes into the PTFE AF polymer. In addition, these interactions can be strongly affected by the $\mathrm{pH}$ of the sample, such as is the case for ionizable molecules (such as11-nor-9-carboxy-D-THC); as such, the reported trends should not be considered universal.

Analytical performance of the PTFE AF/HLB coatings. To test the analytical performance of the PTFE AF/HLB coating in terms of LOQ, as well as both inter-fiber and intra-fiber reproducibility, a group of banned doping substances was selected as model compounds. This choice of model compounds was made in view of the growing interest in monitoring these compounds in biofluids obtained from competing athletes. The achieved results were compared to the minimum required performance limits (MRPLs) set by the World Anti-Doping Agency (WADA) for methods intended for screening of doping compounds. The investigation was carried out in PBS solution in view of its close similarity to urine (no binding components, and $\mathrm{pH}$ usually adjusted to physiological levels), which is the biofluid most often investigated for doping analysis. Accordingly, the LOQ values obtained in PBS can indicate with good approximation if the method developed meets MRPL values set by WADA for urine. In order to determine the LOQ values of the studied compounds for the newly developed coating, a set of calibration solutions within a range of $0.1-1000 \mathrm{ng} \mathrm{mL}^{-1}$ were prepared in PBS. In addition, deuterated internal standards, representative of various physicochemical properties (Table S1), were used for method validation. These internal standards can correct for possible variations between individual fibers, experimental bias, injection variations, and instrumental drifts. The obtained LOQs and regression coefficients $\left(R^{2}\right)$ for the calibrations are reported in Table S2. Considering the most suitable IS (Table 1 ), all tested analytes showed $R^{2}$ values 
higher than 0.992. The LOQ for each analyte was chosen as the lowest calibration level providing less than $20 \%$ deviation from the nominal concentration in the constructed calibration curve, in accordance with FDA directives. The obtained

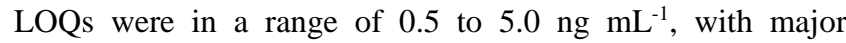
distribution around $1.0 \mathrm{ng} \mathrm{mL}^{-1}$. In comparison to MRPL values set by WADA, only clenbuterol, with an LOQ of $1.0 \mathrm{ng}$ $\mathrm{mL}^{-1}$ versus MRPL of $0.2 \mathrm{ng} \mathrm{mL}^{-1}$, and stanozolol, with an LOQ of $5.0 \mathrm{ng} \mathrm{mL}{ }^{-1}$ versus MRPL of $2.0 \mathrm{ng} \mathrm{mL}-1$, could not meet the set criteria. Considering that the values obtained are in the same order of magnitude as the imposed values, the criteria for these two compounds can be still met by using smaller desorption volumes or more sensitive instrumentation. However, for all other tested compounds, the obtained LOQs were generally orders of magnitude lower than the required MRPLs of WADA. Inter- and intra-fiber reproducibilities were also evaluated (Table S2), and adequate values were generally obtained. It should be kept in mind that the tested coating is only a prototype; further improvement can be accomplished through high throughput production and optimization.

Assessment of extraction capability of fluorocarbon polymer. The selection of a fluorocarbon polymer as a particle immobilizer circumvented the drawbacks associated with other polymeric glues used to date for fabrication of SPME commercial coatings, such as bleeding and long curing times for PDMS, as well as the thermo-lability of PAN. However, it is also important to evaluate the extraction capability of the PTFE AF polymer itself, to assess if its contribution in the extraction process can play a critical role for the coatings investigated. For this purpose, coatings constituted by only PTFE AF were prepared, and extractions performed in solutions containing GC- and LC-amenable analytes (Table S1). The coatings were subsequently desorbed by both thermal and solvent desorption, and the obtained results compared to those achieved at the same working conditions by the PTFE AF/HLB coating. The contribution of the PTFE AF polymer to the extraction was evaluated as the percentage relative to the amount of analyte extracted at the same working conditions by the PTFE AF/HLB coating (Figure S8). The results reveal that the contribution of the PTFE AF polymer to the extracted analyte amounts was not significant (less than $5 \%$ for the majority of the analytes tested). Generally, analytes presenting medium to high hydrophobicity tended to be extracted in larger quantities by the pure PTFE AF coating. Within the LCamenable analytes, 11-nor-9-carboxy-D-THC (log P 5.24), presented the highest extraction efficiency in pure PTFE AF compared to the PTFE AF HLB coating (11\%). Regarding the GC-amenable compounds, $65 \%$ of them were not extracted by the PTFE polymer, with the exception of benzene $(0.2 \%)$, citral $(0.02 \%)$, undecanal (69\%), 2-undecanone (11\%), ethyl nonanoate $(41 \%), \alpha$-pinene (2\%), limonene (13\%), and ethyl undecanoate $(54 \%)$. It is worth noting that this evaluation is purely qualitative in nature, since the amount of polymer in the pure PTFE AF coating was higher than the content present in the PTFE AF/HLB coating.

Preliminary biocompatibility assessment. Owing to their versatility, and as previously mentioned, the coating herein proposed can find a broad variety of applications in metabolomics studies. Consequently, its suitability for direct sampling of plants, animal tissue, and biofluids needs to be further evaluated. For this reason, it is important to assess the biocompatibility of the coating, defined as inertness towards biomaterials. This is a requirement of primary importance in instances where in-vivo or in-vitro extractions are performed by directly exposing the coating to real samples such as tissues or biofluids. Coating biocompatibility is important not only to protect the living tissue exposed to the coating from adverse reactions initiated by contact with the artificial device but also, from an analytical standpoint, to prevent the accumulation of biofouling on the coating surface so as to eliminate bias in the extraction process. To perform a preliminary assessment of coating biocompatibility, whole blood was selected as a model matrix due to its complexity, which usually leads to extensive sample pre-treatment prior to analysis. The coating was tested for consecutive extraction/rinsing/solvent desorption cycles typical of the routine procedures applied for SPME in LC applications. Extractions took place in whole blood for 30 $\mathrm{min}$, at $500 \mathrm{rpm}$, and room temperature. After extraction, the coating was quickly rinsed in ultrapure water (10 sec). Subsequently, desorption was performed for $15 \mathrm{~min}$ in a mixture consisted of $\mathrm{ACN} / \mathrm{MeOH} / \mathrm{H}_{2} \mathrm{O}(30 / 30 / 40, \mathrm{v} / \mathrm{v} / \mathrm{v})$ at $500 \mathrm{rpm}$. Microscopic inspection of the coating was performed to identify whether deposition of blood components on the coating surface occurred. Figure S9 shows the coating surface a) before, b) after the $1^{\text {st }}$ extraction cycle, and c) and d) after 15 consecutive extraction/rinsing/solvent desorption cycles. No accumulation of matrix components was noted to occur after the first extraction, indicating the suitability of these coating as disposable probes for blood and animal tissues sampling. After 15 extractions, the coating could preserve its original conditions; thus its reusability, when possible, could also be considered as an option. Furthermore, absolute matrix effect, defined as ion suppression or enhancement due to coeluting matrix constituents extracted or attached to the coating, was evaluated at two different concentrations (10 and $100 \mathrm{ng} \mathrm{ml} \mathrm{m}^{-1}$ ) in spiked in blood samples. Considering the possibility of using the new SPME coating for single use devices or for multiple extraction, the results were recorded after a single use and 5 consecutive extractions from whole blood samples, under the experimental conditions specified above. The results showed in Table S3 reveal that the chemical composition and morphology of the coating, in conjunction with the cleaning steps applied, effectively avoided the occurrence of ion suppression or enhancement in ESI. To corroborate the hypothesis that no matrix constituents remain attached or accumulate on the coating surface after extraction, scanning electron microscopy/energy dispersive $\mathrm{X}$ ray spectroscopy (SEM/EDX) was performed: the results obtained are shown and discussed in Figure S10 and Section 5 of Supplementary Information. However, a comprehensive assessment of coating biocompatibility in varied complex biomatrices is out of the scope of the present work. On-going studies are being carried out to further investigate the biocompatibility of the coating towards different biomatrices.

Conclusions and future perspectives. This work presents the development of a PTFE AF/HLB coating as a new SPME tool for complementary studies involving gas- and liquidchromatography by means of a single, reliable, and versatile 
extraction phase. The broad range of analytes extractable by HLB particles combined with the inertness of the PTFE AF polymer, employed as a particle immobilizer, was confirmed to be a suitable combination for an SPME coating able to perform multiresidue analysis in complex matrices. In light of this, the new coating can be considered as a suitable candidate for untargeted screening of complex systems; in addition to enhancing the amount of chemical information retrievable due to the extraction capability of HLB particles, the suitability of the coating for such investigations is owed to its aptness for both thermal and solvent desorption, thus enabling the analysis of analytes amenable to different separation platforms. The demonstrated performance of the coating towards the tested GC- and LC-amenable analytes pave the way for possible applications of the coating towards in-vivo studies on plants, animal tissue, and varied biological fluids.

\section{ASSOCIATED CONTENT}

\section{Supporting Information}

The Supporting Information is available free of charge on the ACS Publications website.

Sections 1-5, describing: LC-MS analysis, GC-MS analysis, desorption solvent selection, coating preparation optimization, Scanning electron microscopy/energy dispersive X-ray spectroscopy (SEM/EDX) analysis. Figure S1-10. Tables S1-3.

\section{AUTHOR INFORMATION}

\section{Corresponding Author}

*Tel.:+1-519-888-4641. Fax: + 519746 0435. E-mail: janusz@ uwaterloo.ca.

\section{Author Contributions}

All authors have given approval to the final version of the manuscript.

\section{Notes}

The authors declare no competing financial interest.

\section{ACKNOWLEDGMENT}

The authors thank Dr. John Vukovic from Waters Corporation for providing the HLB particles used for this study and Supelco/Sigma Aldrich for the C18/SCX/PAN fibers and the LC column.

\section{REFERENCES}

(1) Souza-Silva, É. A.; Jiang, R.; Rodríguez-Lafuente, A.; Gionfriddo, E.; Pawliszyn, J. TrAC Trends Anal. Chem. 2015, $71,224-235$.

(2) Souza-Silva, É. A.; Gionfriddo, E.; Pawliszyn, J. TrAC Trends Anal. Chem. 2015, 71, 236-248.

(3) Souza-Silva, É. A.; Reyes-Garcés, N.; Gómez-Ríos, G. A.; Boyaci, E.; Bojko, B.; Pawliszyn, J. TrAC Trends Anal. Chem. 2015, 71, 249-264.

(4) Bojko, B.; Reyes-Garcés, N.; Bessonneau, V.; Goryński, K.; Mousavi, F.; Souza Silva, E. A.; Pawliszyn, J. TrAC Trends Anal. Chem. 2014, 61, 168-180.

(5) Mousavi, F.; Gionfriddo, E.; Carasek, E.; Souza-Silva, E. A.; Pawliszyn, J. Metabolomics 2016, 12, 169.

(6) Musteata, M. L.; Musteata, F. M.; Pawliszyn, J. Anal. Chem.
(7) Souza-Silva, E. A.; Pawliszyn, J. Anal. Chem. 2012, 84, 69336938.

(8) Thompson, E. V. J. Polym. Sci. Part B Polym. Lett. 1966, 4, 361-366.

(9) O’Neal, K. L.; Zhang, H.; Yang, Y.; Hong, L.; Lu, D.; Weber, S. G. J. Chromatogr. A 2010, 1217, 2287-2295.

(10) Lu, D.; Weber, S. J. Chromatogr. A 2014, 1360, 17-22.

(11) Zhang, H.; Weber, S. G. Top. Curr. Chem. 2012, 308, 307-337.

(12) Lu, D.; Weber, S. J. Chromatogr. A 2014, 1360, 1-6.

(13) Polyakov, A. J. Memb. Sci. 2003, 216, 241-256.

(14) Zhang, H.; Wang, S.; Weber, S. G. Anal. Chem. 2012, 84, 9920 9927.

(15) Zhang, H.; Wang, S.; Weber, S. G. J. Memb. Sci. 2013, 443, 115-123.

(16) Zhao, H.; Zhang, J.; Wu, N.; Zhang, X.; Crowley, K.; Weber, S. G. J. Am. Chem. Soc. 2005, 127, 15112-15119.

(17) Nishida, K.; Sakaguchi, H.; Xie, P.; Terasawa, Y.; Ozawa, M.; Kamei, M.; Nishida, K. J. Artif. Organs 2011, 14, 357-363.

(18) Reyes-Garcés, N.; Bojko, B.; Hein, D.; Pawliszyn, J. Anal. Chem. 2015, 87, 9722-9730.

(19) Reyes-Garcés, N.; Bojko, B.; Pawliszyn, J. J. Chromatogr. A 2014, 1374, 40-49.

(20) Bessonneau, V.; Boyaci, E.; Maciazek-Jurczyk, M.; Pawliszyn, J. Anal. Chim. Acta 2015, 856, 35-45.

(21) Merkle, S.; Kleeberg, K.; Fritsche, J. Chromatography 2015, 2, 293-381.

(22) Buchholz, K. D.; Pawliszyn, J. Anal. Chem. 1994, 66, 160-167.

(23) Grandy, J. J.; Boyacı, E.; Pawliszyn, J. Anal. Chem. 2016, 88, 1760-1767.

(24) Risticevic, S.; DeEll, J. R.; Pawliszyn, J. J. Chromatogr. A 2012, 1251, 208-218.

(25) Pawliszyn, J. In Handbook of Solid Phase Microextraction; 2012; pp. 13-59.

(26) Górecki, T.; Yu, X.; Pawliszyn, J. Analyst 1999, 124, 643-649.

(27) Souza-Silva, É. A.; Gionfriddo, E.; Alam, M. N.; Pawliszyn, J. Anal. Chem. 2017, acs.analchem.6b04442.

(28) Polyakov, A.; Starannikova, L. E.; Yampolskii, Y. P. J. Memb. Sci. 2003, 216, 241-256.

(29) Bojko, B.; Reyes-Garcés, N.; Bessonneau, V.; Goryński, K.; Mousavi, F.; Souza Silva, E. A.; Pawliszyn, J. TrAC Trends Anal. Chem. 2014, 61, 168-180.

(30) Bessonneau, V.; Bojko, B.; Pawliszyn, J. Bioanalysis 2013, 5 783-792.

(31) Vuckovic, D.; de Lannoy, I.; Gien, B.; Shirey, R. E.; Sidisky, L. M.; Dutta, S.; Pawliszyn, J. Angew. Chemie 2011, 123, 54565460.

(32) Bojko, B.; Vuckovic, D.; Pawliszyn, J. J. Pharm. Biomed. Anal. 2012, 66, 91-99.

(33) Bojko, B.; Gorynski, K.; Gomez-Rios, G. A.; Cudjoe, E.; Pawliszyn, J. Anal. Chim. Acta 2013, 803, 75-81.

(34) Bojko, B.; Wąsowicz, M.; Pawliszyn, J. J. Pharm. Anal. 2014, 4, 6-13. 


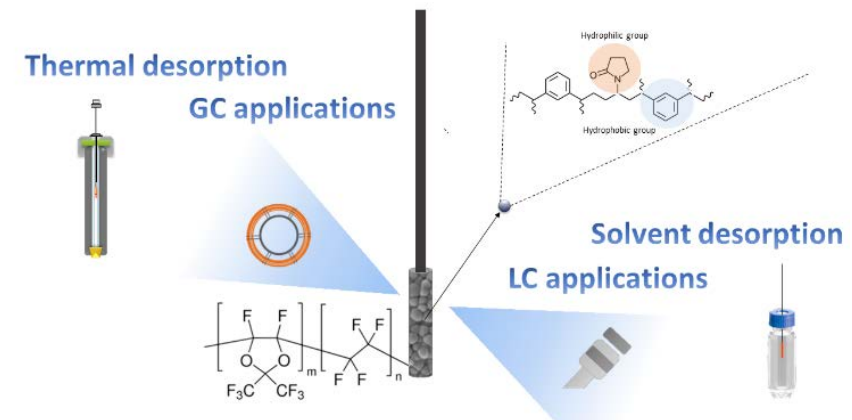

